\title{
The Psychobiology of Hunger - A Scientific Perspective
}

\author{
Kristine Beaulieu' ${ }^{1} \cdot$ John Blundell ${ }^{1}$
}

Accepted: 26 September 2020 / Published online: 6 October 2020

(c) The Author(s) 2020

\begin{abstract}
From a scientific perspective, hunger can be regarded as an identifiable conscious sensation which can be distinguished from other conscious states (e.g., pain, fear). The hunger state can be measured and is a marker of the existence of underlying biological processes. Measured hunger is functional and is normally associated with the act of eating. However, the conscious state of hunger, although driven physiologically, is not exclusively determined by biology; there is an environmental influence that can modulate its intensity and periodicity, and cultural factors that shape the appropriateness of the expression of hunger. Within a psychobiological framework, hunger can be considered as the expression of a 'need state' which mediates between biological requirements and environmental (nutritional) satisfaction. Our empirical studies show that hunger is clearly associated with biological signals (e.g., resting metabolic rate and some gastrointestinal peptides) and is central to the relationship between energy expenditure and energy intake.
\end{abstract}

Keywords Appetite control $\cdot$ Energy balance $\cdot$ Eating behaviour $\cdot$ Empirical studies $\cdot$ Human physiology

\section{Orientation}

This manuscript will set out a scientific perspective on hunger. This approach is not infallible but is dependent on a scientific method that can be broadly applied in physics, biology, and psychology. The process is normally quantitative and objective, and the understanding (of the world) is arrived at by measuring, recording, and analysing happenings-usually producing a material output referred to as data. The statistical analysis and graphical representation of these data form the basis of scientific evidence. These data then have to be subjected to interpretation and logical reasoning in order to lead to the most veridical and trustworthy understanding. The beauty of science is that the evidence and logical reasoning can be challenged. In this way, the scientific method arrives at a state of optimal understanding by a process of refinement. In this paper, we endeavour to show the way in which these processes lead to a true (or approximately true) understanding of hunger.

Psychology's field of study is composed of millions of human beings who are biologically quite similar but socially

Kristine Beaulieu

k.beaulieu@leeds.ac.uk

1 School of Psychology, Faculty of Medicine and Health, University of Leeds, Leeds LS2 9JT, UK and culturally extremely diverse. Conscious sensations (such as hunger) and behavioural actions (such as eating) display enormous heterogeneity. It is widely regarded that hunger is a conscious sensation experienced by every human being on the planet; it is a universal human experience. However, this does not mean that it is experienced by every human being in exactly the same way. This forms a further part of the intention of this scientific perspective; to recognise how scientific methods can reach an understanding of hunger in which the 'scientific evidence' reflects individuality and can be discussed using a common language.

Initially, we wish to point out that we are scientists and not philosophers and hence it is not possible to provide a profound philosophical justification for the framework adopted. However, it is necessary to note that our approach to scientific research is not arbitrary or casual. Our view - as scientists - is based on the premise that the goal of science is to arrive at a true, or approximately true, description of reality. This view coincides with that outlined by, among others, Sokal (2008) and can be extended to incorporate the aim to 'obtain an objective (albeit approximate and incomplete) understanding of (some aspects) of the world' (Sokal 2008, p. 270). By extending our view to incorporate the goal of reaching an understanding of the world, we recognise that this issue has been elegantly adumbrated by Potochnik (2017). 


\section{Function of Hunger}

From a scientific perspective, hunger can be regarded as an identifiable conscious sensation which can be distinguished from other conscious states (e.g., pain, fear, tiredness, etc.) (Blundell 1979). Hunger is a biologically useful sensation which reminds organisms that food should be sought or eaten. Hunger is primarily a biologically instigated drive for food that is essential for life. Hunger can therefore be regarded as a quantifiable biological trait of humans that is a predictor of the willingness to eat and of the amount of food to be eaten (Stubbs et al. 2000). For many reasons this predictive power is not perfect, nor should it be expected to be. Hunger is neither a necessary nor a sufficient condition for eating; but it is a strong risk factor. People are more likely to eat if they are in a state of hunger than if they are not. However, in the current obesogenic food environment, the contribution of hedonic- or reward-driven food intake to obesity cannot be ignored (Berthoud et al. 2017). Therefore, it is important to distinguish between eating driven by hunger and eating prompted by a desire for the pleasurable taste of food. This distinction is often described as hunger vs pleasure, or homeostasis vs hedonics. Neither of these dyads is completely accurate but they serve to highlight an important issue.

In the context of the psychobiological framework of appetite, homeostatic processes link the physiological needs for energy with behaviour that satisfies theses needs (Hopkins et al. 2017). These processes are shaped by excitatory and inhibitory signals. In contrast, hedonic appetite includes thoughts about food and sensory appreciation of certain food attributes that determine food preferences and choice, and is reflected by liking and wanting for food (Finlayson et al. 2007). Within this framework, hunger is considered as a powerful mental entity that reflects a biological need state (Caudwell et al. 2011). Our studies show that the state of hunger is clearly associated with biological signals (e.g. resting metabolic rate and some gastrointestinal peptides) (Gibbons et al. 2013; Hopkins et al. 2016, 2018). Hunger is also central to the relationship between energy expenditure and energy intake; hunger reflects the degree of a person's physical activity (Beaulieu et al. 2018; Hopkins et al. 2019).

\section{Objectivity and Quantification of Hunger}

How is it possible to operationalise a subjective conscious sensation for the purpose of scientific research? It is extremely important that the intensity of perceived hunger can be quantified through a process that translates a conscious sensation into a physical action (Flint et al. 2000; Stubbs et al. 2000). This translation process is based on the principle of the visual analogue scale (VAS), which is a key component in a psychophysical procedure that requires a person to estimate the strength of the sensation of hunger currently being experienced, and to represent this as a point on a single line on a screen or a piece of paper (hence VAS). The line normally has labelled end points which are 'no feeling of hunger at all' to 'as hungry as I have ever felt'. The person makes a judgement of the current strength of their experience relative to these two extreme positions. This procedure represents an interval scale (there are other types of psychophysical scales) and the selected point on the line (normally $100 \mathrm{~mm}$ in length) is assigned a numerical value. Figure 1 demonstrates an example of how hunger can be measured and recorded at regular intervals during the day using a hand-held electronic appetite rating system (EARS) incorporating a VAS (Gibbons et al. 2011). This can also be achieved by traditional pen and paper methodology. The data collected can then be processed statistically in the same way as other scientific variables and can be used to evaluate the effects of different foods, physiological states, behavioural conditions, environmental stimuli, and certain disease states on hunger.

\subsection{Validity}

This quantification of hunger has certain psychophysical properties which allow it to be used in a functional way. For example, the common-sense view is that hunger is a prelude to eating food. People use the word hunger in this context, and it is part of a shared cultural experience that shapes the identity of hunger. In order that hunger can be used in this way, and can be meaningfully used, it has to be demonstrated that hunger bears some invariant relationship to the pattern of eating. Figure 2 illustrates how the perceived intensity of hunger varies across the day in relation to meals consumed. The perceived intensity of hunger tracks the pattern of meals across the day, reaching a zenith before each meal and falling to a nadir at the end of a meal. The interpretation is that

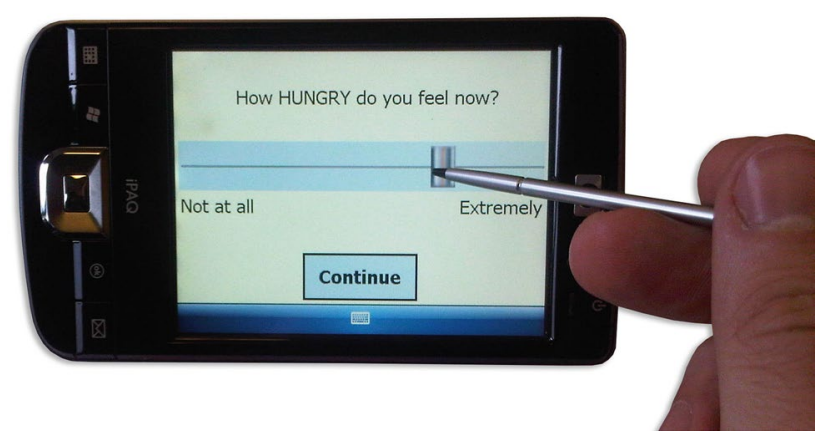

Fig. 1 Example of an electronic appetite rating system using a visual analogue scale (VAS) to quantify perceived hunger (Gibbons et al. 2011). Adapted from Gibbons et al. (2014) 


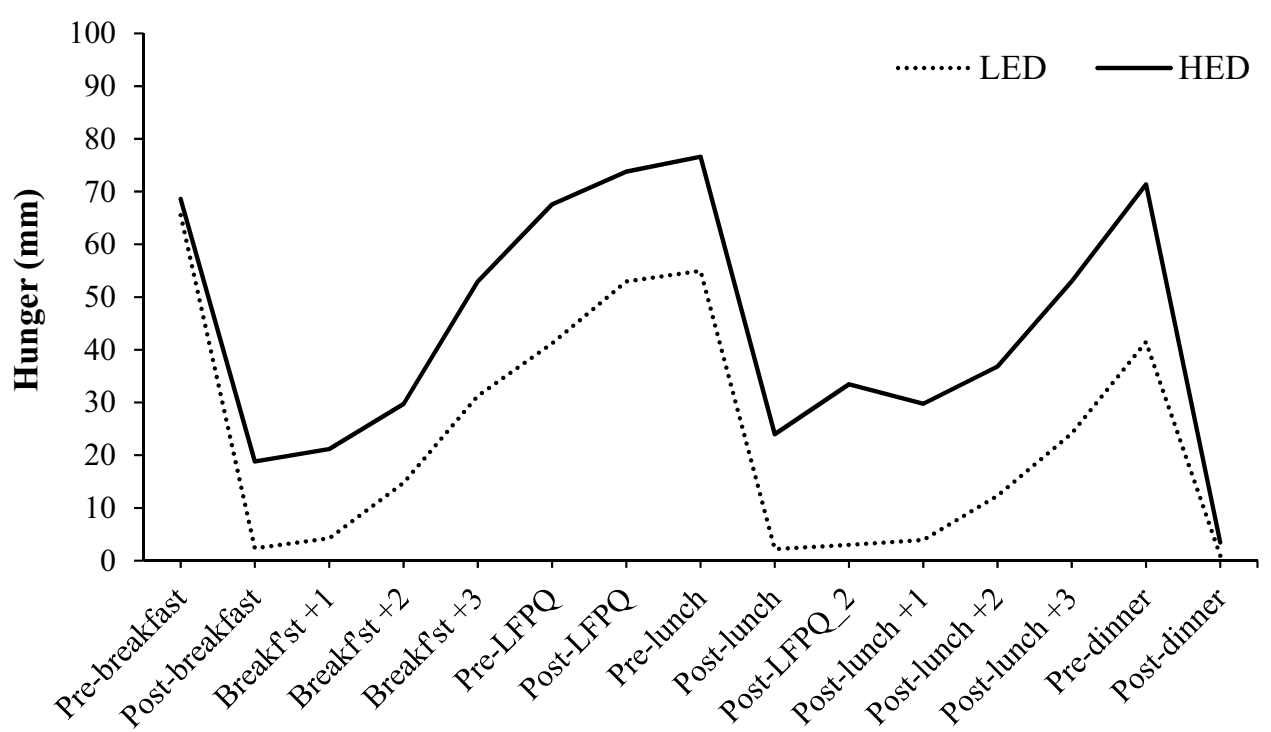

Time point

Fig. 2 Mean variation in subjective hunger ratings in response to low-energy-dense (LED; $\leq 0.8 \mathrm{kcal} / \mathrm{g}$ ) and high-energy-dense $(\mathrm{HED} ; \geq 2.5 \mathrm{kcal} / \mathrm{g}$ ) food intake throughout an 8-h laboratory probe day (measured with a 100-mm visual analogue scale on an electronic hand-held device, the EARS) in women with overweight or obesity (adapted from Buckland et al. 2018). As shown, hunger is suppressed in response to food intake, and slowly increases over the post-meal interval, until the following meal. The nutritional manipulation of the consumed meals also shows that hunger is also more suppressed in response to low-energy-dense compared to high-energy-dense foods some biological signal stimulates the sensation of hunger which is, in turn, transiently suppressed by the consumption of food. This figure also shows that some factor associated with the ingestion of food modulates the degree of perceived hunger. Some foods (of low energy density: small number of calories for a given weight or volume of food) have a much greater suppressive effect on hunger than foods of high energy density (Rolls 2017; Buckland et al. 2018). Lowenergy-dense foods include fruits, vegetables and generally high-water and high-fibre items, whilst high-energy-dense foods are usually high in fat. Indeed, there are many examples of the perceived intensity of hunger being modulated by physical and nutritional characteristics of the consumed food (e.g. weight, volume, energy content or energy density). This is an important feature of the 'Satiety Cascade' (see below).

\subsection{Individual Heterogeneity}

Observing the profiles illustrated in Fig. 2, it is easy to deduce that a person's perceived hunger follows a smooth trajectory across the day, and that everyone's perceptions are identical. This is not the case; hunger is a phenomenon that reflects individual differences. An important feature of these smooth hunger profiles is that they are based on the group average of individual values. However, as indicated in Fig. 3, an individual's perception of hunger does not conform to the average value. This leads to an important methodological consideration. Since individual perceptions of hunger vary greatly, it is important in research studies to use 'within subject' designs in which each person is used as their own comparison under two or more conditions.

Note: It is important to distinguish between two features of this account of hunger. First is the demonstration that hunger can be regarded as a scientific entity which can be measured and quantified. This establishes that hunger can be used in scientific investigations. A separate feature concerns the nature of the processes that influence hunger-biological, personal or environmental. These mechanisms are also part of the scientific approach. The examination of such mechanisms - that deal with the identity and function of hunger-becomes more coherent if there is a clear way of measuring hunger. However, the measurement of hunger is separate from the mechanisms through which it is aroused and suppressed.

\section{Hunger and the Satiety Cascade}

Both in the field of science and in day-to-day activities, hunger is intimately associated with the consumption of food. Indeed, in common language, hunger is used to account for the act of eating. This is exemplified by expressions such as: 'I'm hungry, I need something to eat'. In science, hunger reflects this power of the need to eat as part 
of a psychobiological system whose components interact to influence the moment-to-moment perception of hunger. In both instances, hunger can be understood in relation to the concept of the Satiety Cascade. In this regard, hunger is often used in association with the terms satiation and satiety, which themselves are key features of the Satiety Cascade, and it is important to distinguish these terms (Blundell et al. 2010). Let's agree that hunger is the drive to consume; eliciting and sustaining a behavioural response (eating) to a biological need. After eating has been initiated, satiation is the process that leads to meal termination (i.e. controls meal size) and satiety is the process that inhibits further eating (i.e. post-meal suppression of hunger) (Blundell et al.
2001). This conceptual scheme called the Satiety Cascade was proposed over 30 years ago (Blundell et al. 1987) and can be used to illustrate the relationship between hunger and the pattern of eating (Fig. 4). Hunger is therefore conceptualised as part of a broader system of appetite control. The rise and fall of hunger during the day drives patterns of food intake. Hunger initiates a meal while satiety signals - arising from the physiological responses to eating during a meal and the post-ingestive period - suppress hunger and help to determine how much is eaten and how much time will elapse before the next meal. In this context, it is important that satiety is often defined by an inverse relationship with the perceived intensity of hunger; when hunger is high, satiety
Fig. 3 Individual variability in the hunger response $(100-\mathrm{mm}$ visual analogue scale) to a 9-h laboratory probe day in a selection of non-obese adults (sample reduced for clarity of the figure) from Beaulieu et al. (2017). Participants consumed an individually calibrated breakfast (25\% of resting metabolic rate; $0 \mathrm{~h})$, a $700-\mathrm{kcal}$ snack $(3 \mathrm{~h})$ and an ad libitum lunch and dinner ( $4 \mathrm{~h}$ and $8 \mathrm{~h}$, respectively), where they were asked to eat as much or as little as they wanted until comfortably full

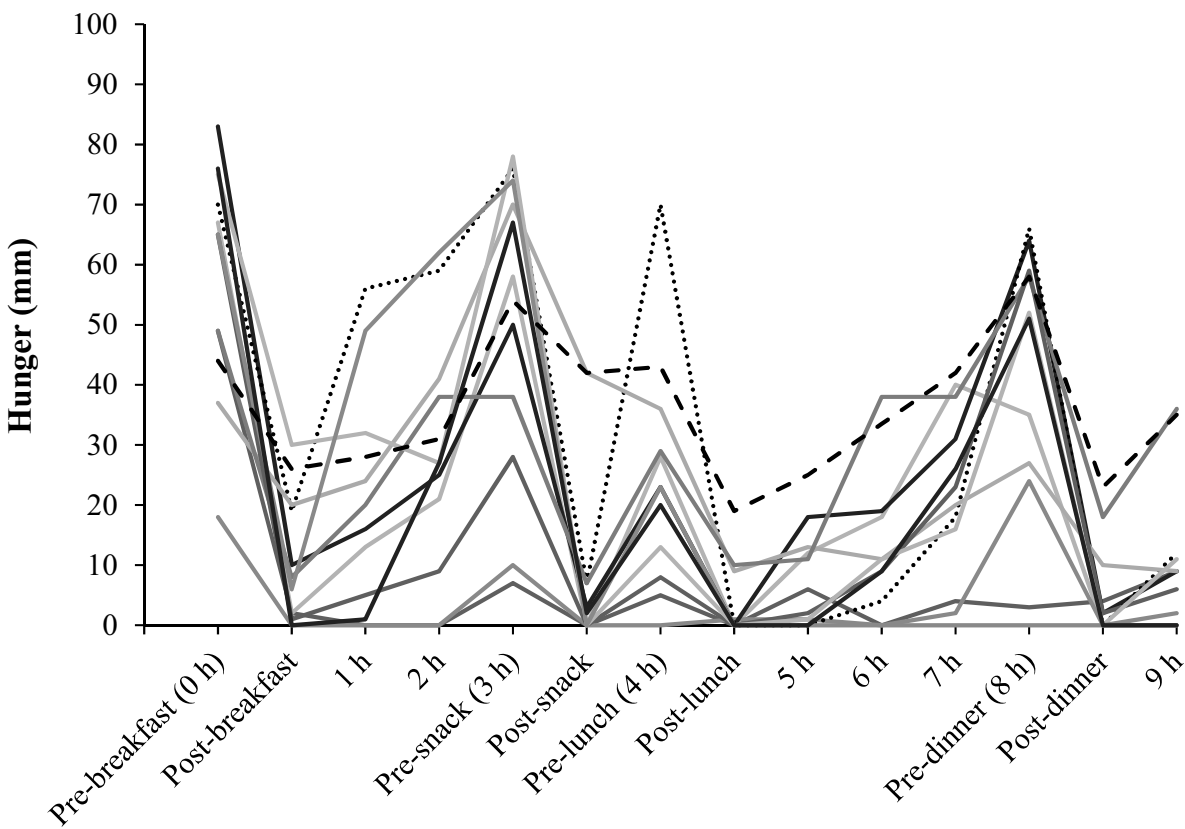

Time point

Fig. 4 Conceptualisation of the Satiety Cascade. Hunger drives food intake, which triggers a series of signals which terminate food intake (satiation) and lead to the post-meal suppression of hunger (satiety). These homeostatic processes interact with hedonic processes in the overall control of appetite and food intake. Adapted from Blundell and Finlayson (2008)
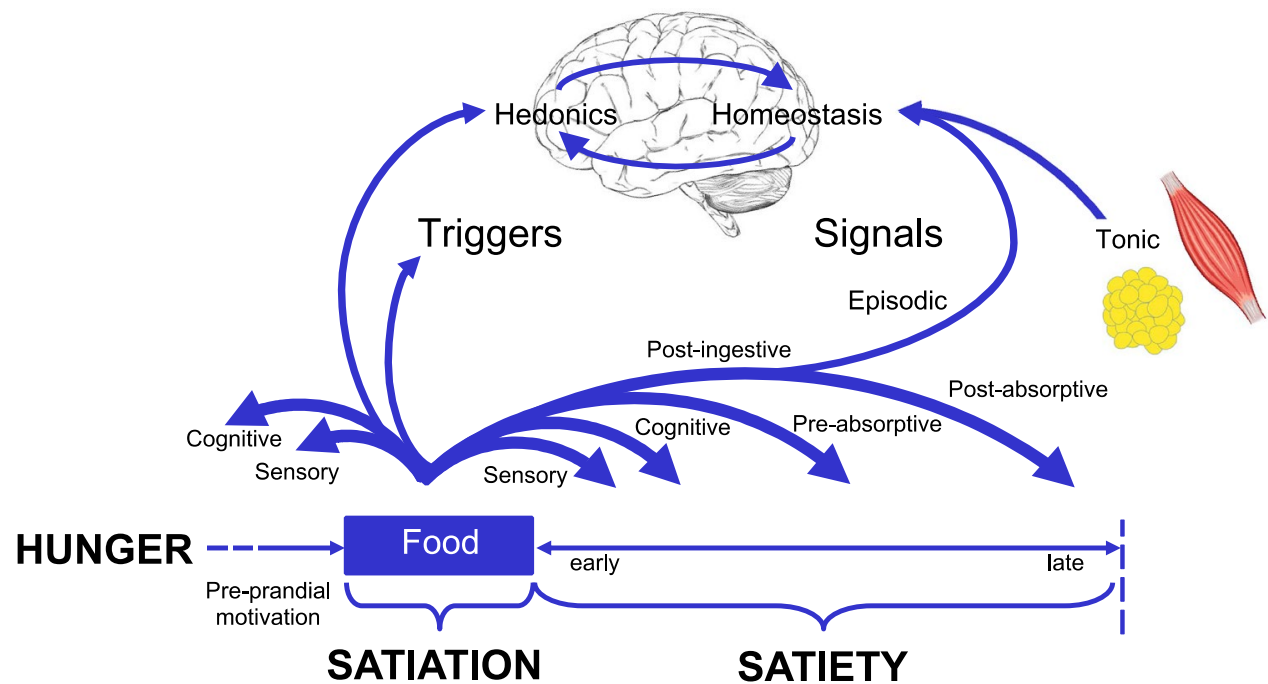
is weak and vice versa. Although it should be remembered that satiety is measured according to the degree of hunger experienced.

\subsection{Interaction Between Hunger and Food Hedonics}

In addition to the hunger arising from biological needs, sensations resembling hunger, sometimes termed 'hedonic hunger' can be generated externally from the taste and thoughts of foods (Lowe and Butryn 2007). The motivation towards and desire to eat certain food stem from the hedonic appetite system and interact with the homeostatic appetite system, as shown in Fig. 4. These hedonic signals may override homeostatic satiety signals and constitute one cause of overconsumption of food (Erlanson-Albertsson 2005). This can be reflected in both satiation (by increasing the size of a meal) and satiety (by eating in the absence of hunger during the post-ingestive period).

Note: An important question to pose here is whether 'hedonic hunger' really has the characteristics of a biological drive and is appropriately termed hunger? Is 'hedonic hunger' simply a reflection of cognitive impulses motivating the desire to eat or the attraction towards certain foods, thus actually representing a form of implicit wanting? While these two processes may be difficult to separately identify when casually observing a person eating, it is important to make the distinction between hunger (biological drive) and implicit wanting (component of the hedonic system), as separate, but interacting, entities. Hunger as a biological drive is further discussed below.

\section{Hunger Reflects a State of Biological Need}

There is evidence that hunger is associated with fundamental biological processes which throw light on the function of hunger and its evolutionary purpose. From an evolutionary perspective, there is good reason to propose that the drive to eat should arise from the need to replace the energy used by the body, and should be directed to materials in the environment that supply energy and other nutrients (namely foods). Surprisingly, this concept has been slow to become accepted in biology (due to the strong belief in the principle of endogenous regulation) even though the basic idea was proposed more than 60 years ago (Edholm et al. 1955, 1970; Mayer et al. 1956). There is a clear logic to the idea that energy used by the body should be a major factor in driving energy intake. This energy demand in the body is represented by the resting metabolic rate (RMR) which is a measure of the energy used to maintain bodily tissues and to support vital biological functions. It follows that RMR must be dependent on the structure of the body (or body composition) since metabolism is generated by cellular activity. In a basic twocompartment model of body composition, lean mass (or fatfree mass; FFM) can be distinguished from fat mass (FM). FFM uses much more energy for maintenance than FM. Therefore, FFM exhibits a much larger effect on RMR than FM. Important studies demonstrated that FFM was strongly associated with voluntary energy intake (eating) but FM was not (Fig. 5a, b). In turn, it has been demonstrated that the metabolic drive (RMR) is strongly associated with the perception of hunger (Fig. 5c). Importantly, FFM includes all high-metabolic-rate organs of the body (heart, lungs, liver, kidneys, brain, etc.) which contribute hugely to RMR.

Note: This account illustrates why hunger is a strong biological drive whose function can only be denied under peculiar conditions (e.g. anorexia nervosa or as a volitional political or religious act). Hunger is a fundamental life force. From an evolutionary perspective, hunger is functional and reflects the body's energy requirements for maintaining vital organs (heart, liver, brain, kidney, muscle, etc.). Hunger can be considered as the expression of a 'need state' which mediates between biological requirements and environmental (nutritional) resources (foods).

\section{Hunger as a Component of an Appetite System: Tonic and Episodic Processes}

It is part of our theoretical view that, in human biology and psychology, individual traits, processes, or abilities cannot be understood in isolation. This is true for hunger which, from a scientific perspective, can only be fully appreciated as part of a system. This systemic position is described here. A feature of the scientific approach to hunger is that it is incorporated in physiological processes that integrate the body's biology with the nutritional environment. Fundamental to this principle is the proposition that hunger is a measurable manifestation of the drive to eat and forms part of what we can identify as an appetite system (e.g. Blundell 1991). In turn, processes that influence the expression of appetite can be separated into tonic and episodic (Blundell et al. 2001). Tonic processes are rather stable and enduring, and change slowly over time. Episodic processes are organised around the occurrence of episodes of eating behaviour (meals, snacks, etc.); they occur periodically and are shortlived rather than long-lasting. These episodes take various forms within any one day and may change from day-to-day.

Note: The origin of the drive to eat - as described above - is clearly a tonic process and reflects a constant need for energy (arising from the body's continuous use of energy). In humans, this drive is peri- 

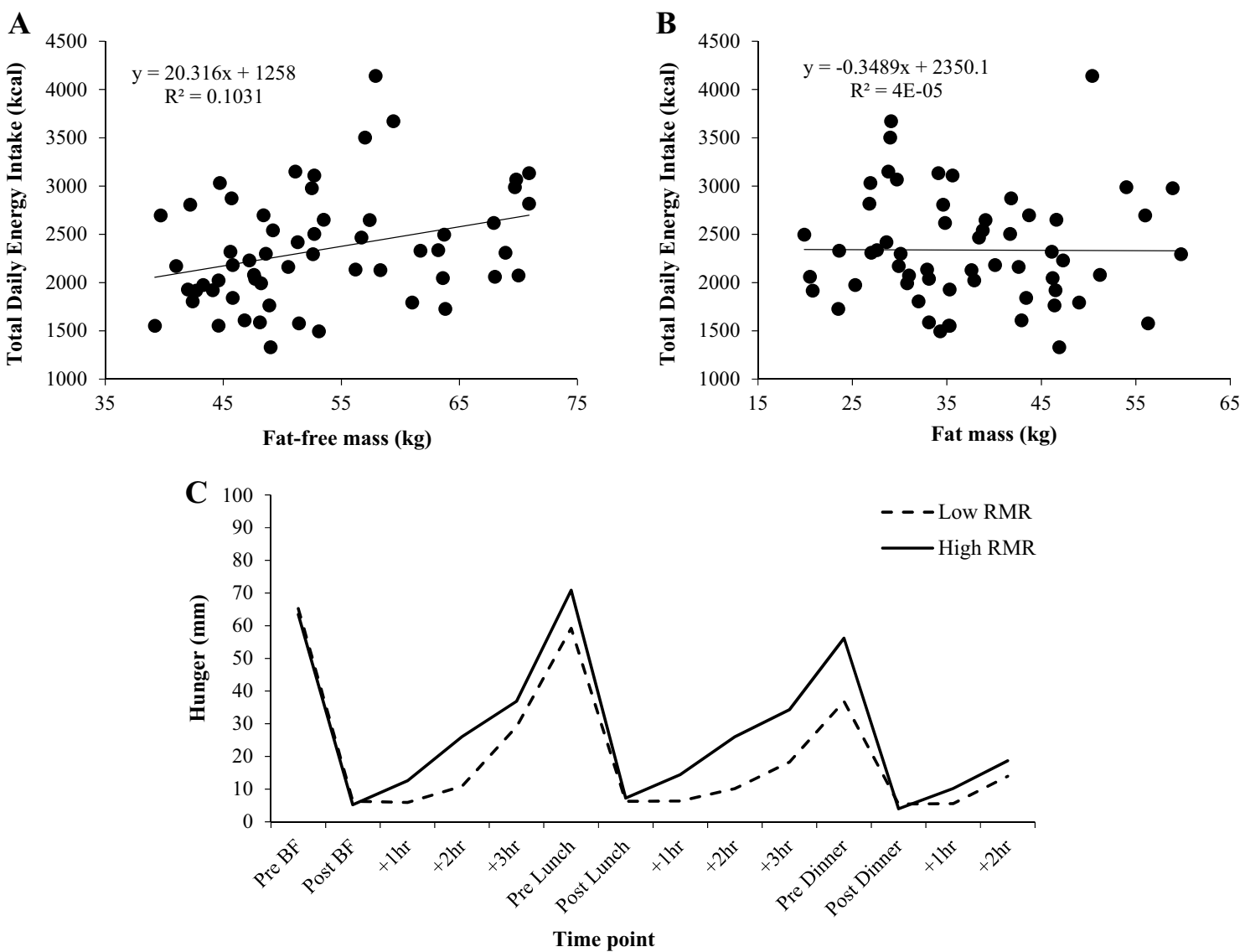

Fig. 5 Relationship between fat-free mass (a) and fat-mass (b) and total daily energy intake measured in the laboratory with a realistic meal pattern based on an ad libitum breakfast, fixed lunch (800 kcal), and ad libitum dinner and evening snack box in adults with overweight or obesity (adapted from Blundell et al. 2012). At the ad libitum meals, participants were asked to eat until comfortably full, and foods were covertly weighed before and after consumption to determine energy intake. As shown in panels a and $\mathbf{b}$, fat-free mass was associated with food intake, whereas there was no association with fat mass. This demonstrates that fat-free mass exerts a strong influence on the drive to eat and hunger. Panel $\mathbf{c}$ illustrates subjective hunger ratings (100-mm visual analogue scale) over the course of one day in response to an ad libitum breakfast, fixed lunch (800 kcal) and ad libitum dinner in adults with overweight or obesity according to upper (high RMR) and lower (low RMR) tertiles stratified by sex (adapted from Caudwell et al. 2013). The figure clearly shows that those participants with a higher RMR (basal metabolic use of energy) had significantly higher levels of hunger throughout the day compared to those with lower RMR. This indicates that the expression of hunger is biologically driven through the energy expended by metabolically active tissues odically released (or triggered) and leads to episodic outputs of eating behaviour (usually called meals or snacks); the drive itself is not episodic (it is constant). This behaviour includes food seeking, acquisition, and eating. The behavioural output is expressed in a particular environment with a specific culture, geographical location, and socio-economic circumstances. These external forces shape the behaviour and determine its structure and form; they determine the foods eaten, the way they are consumed, where and with whom they are eaten, their frequency, and over which period of time. These episodes of behaviour constitute the phenomenon that people recognise as appetite. This episodic appetite behavour is culturally dependent; the tonic drive to eat is independent of culture and is dependent on biological processes. Only when the drive to eat erupts into the external environment as behaviour does the impact of culture become apparent.

Figure 6 illustrates how tonic and episodic influences on hunger can interact. Tonic excitatory processes arise from metabolically active tissues, largely FFM. Tonic inhibitory signals arise from FM (most obviously through the action of the hormone leptin), but are weakened at higher levels of adiposity, which we argue is due to reported leptin resistance (Blundell et al. 2020). This may promote overconsumption and further weight gain in individuals with obesity. In contrast to the tonic processes, episodic inhibitory processes 
vary in strength throughout the day, in response to the pattern of food intake. These inhibitory processes are widely believed to arise from physiological signals in the gastrointestinal tract and include hormones such as cholecystokinin (CCK), peptide YY (PYY) and glucagon-like peptide-1 (GLP-1). Some hormones, such as the so-called 'hunger stimulating hormone' ghrelin, has both episodic and tonic properties. On an episodic level, ghrelin concentrations rise before meals and are suppressed in response to food intake. It has been proposed that this indicates that ghrelin is a trigger for the release of hunger (Blundell et al. 2020). Episodic processes interact with tonic processes such that satiety signalling periodically interrupts the underlying drive to eat.

\section{Hunger is Driven by Total Energy Expenditure}

As noted throughout this text, the function of hunger serves the purpose of reminding us that food should be sought and ingested. Hunger is the perceived motivation that allows the energy needs of the body's metabolism to be replaced through the acquisition of food. In other words, hunger can be represented as a metabolic drive. However, a person's energy needs also arise from the energy used in being physically active and in performing essential daily behavioural tasks. This activity-based energy expenditure also constitutes a drive for energy. An extreme example of this is that of Tour de France cyclists, whose very high food intake is required to balance the extremely high daily energy expenditure (Saris et al. 1989). Consequently, hunger can be regarded as a functional response to energy requirements of both metabolism and behaviour.

This proposition has led to a field of scientific enquiry about the relationship between physical activity and hunger. One currently significant reason behind this is the interest in the relationship of obesity to physical activity and sedentariness. The issue is both important and urgent; and is of major interest for public health. For this reason, a summary of the science is included here. First, does habitual physical activity energy expenditure lead people to experience more hunger? And second, does being sedentary (inactive) take away hunger? One approach is to study people in the natural state who are either habitually active or habitually highly sedentary. Another approach is to study the response of inactive people subjected to an exercise intervention.

Current research had an interesting starting point based on a famous field study carried out more than 60 years ago.

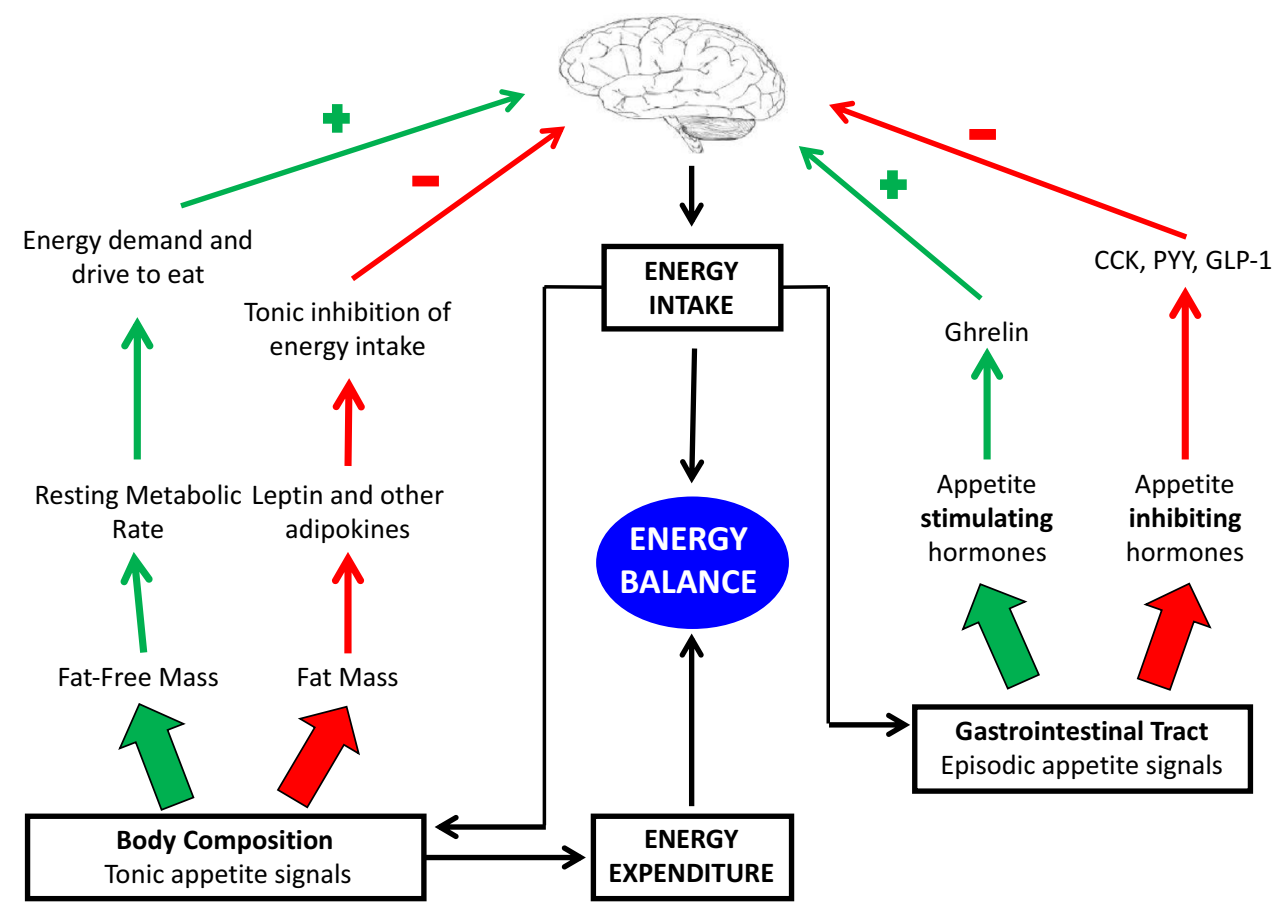

Fig. 6 Tonic and episodic processes of appetite control within a psychobiological framework of appetite. Tonic processes are relatively stable over days and stem from fat-free mass and fat mass. In contrast, episodic processes respond to the presence (or absence) of nutrients in the gastrointestinal tract. Both tonic and episodic processes are comprised of signals that are excitatory (green arrows) or inhibitory (red arrows). Generally, hunger arises through tonic processes and is suppressed by episodic processes; the overall strength of the drive to eat is the balance between these physiological processes. Ultimately, an individual's energy balance (and body weight) will depend on the balance between energy intake and energy expenditure. CCK, cholecystokinin; PYY, peptide YY; GLP-1, glucagon-like peptide-1. Adapted from Blundell et al. (2015). 
The study had a real-life setting and examined the associations among physical activity, food intake and body weight in workers in Calcutta, India (Mayer et al. 1956). The investigators classified jute mill workers into categories of occupational physical activity ranging from 'sedentary' (e.g. stallholders, supervisors) to 'very heavy work' (e.g. blacksmiths, cutters, carriers). They then assessed daily food intake by dietary interviews. Interestingly, they revealed a non-linear (U-shaped) relationship between occupational physical activity level and energy intake. In the workers with higher levels of occupational physical activity (e.g. 'medium' to 'very heavy' work), daily energy expenditure and energy intake were closely matched, but in those with lower levels of occupational physical activity, this coupling was lost, such that daily energy intake exceeded expenditure in the 'sedentary' to 'light' activity workers. Additionally, those in the sedentary category were heavier than those in the light to very heavy work categories (Mayer et al. 1956). This U-shaped relationship between physical activity level (i.e. daily energy expenditure) and energy intake suggests that higher levels of physical activity are associated with more sensitive appetite control (greater hunger and enhanced satiety signalling) and an appropriate 'matching' between energy expenditure and energy intake (first question). In contrast, lower levels of physical activity (i.e. being inactive/highly sedentary) are associated with dysregulated appetite and a reduction of energy expenditure which does not downregulate hunger and food intake (second question). Consequently, this results in overconsumption relative to energy requirements and weight gain. Several studies have now shown that physical activity improves body composition by increasing FFM and decreasing FM, and also influences the release of gastrointestinal hormones involved in satiety signalling (Blundell et al. 2015). Physical activity therefore affects both the tonic and episodic processes of appetite shown in Fig. 6. Additionally, greater levels of habitual physical activity are associated with a greater drive to eat through an independent effect on total daily energy expenditure (Hopkins et al. 2019).

Does this mean that exercise promotes hunger and therefore drives up food intake, in turn leading to overconsumption and obesity? The answer is 'no' because the drive to eat is functional and seeks to replace the energy used. Overconsumption would be caused by the injudicious ingestion of inappropriate high-energy-dense foods. In addition, stimulating physiology through exercise exerts a 'dual-process action' on appetite behaviour (King et al. 2009). This is marked by an increase in the hunger experienced in the early morning but also by an intensification of the satiety response to eating. Physical activity enhances satiety signalling by an influence on the episodic part of the appetite system shown in Fig. 6. For further discussion of this interesting issue see reviews by Blundell et al. (2015) and Beaulieu et al. (2018).
Importantly, and similar to the effect of dietary variables (Fig. 3), the influence of physical activity on hunger also shows substantial individual variability.

The investigation of the intimate relationship between physical activity behaviour and hunger again illustrates how the existence of a reliable and valid objective measurement of hunger can be used to study an important public health problem in today's obesogenic world.

\section{Omothetic and Idiographic Principles}

From a scientific perspective, hunger should not be considered a vague sensation subject to a variety of interpretations. Hunger can be analysed scientifically and it can be quantified. It can be clearly differentiated from other conscious sensations, but is closely related to feelings such as the desire to eat and, inversely, to the feeling of fullness (Stubbs et al. 2000). When measured periodically across the day in people using tracking technology, hunger shows a rhythm with variable amplitude usually synchronised to the pattern of eating (see Fig. 1). The robustness of this pattern allows hunger to be used as a nomothetic principle that gives rise to general rules underlying human behaviour. However, such rules are not absolute, and they are not perfect. Therefore, they should not be expected to operate with exact precision. It is important to remember that psychobiology is not physics, and when we deal with human behaviour and conscious experiences, even though they can be quantified, they remain subject to a huge number of possible influences. Hunger profiles also display substantial individual variability reflecting biological (and psychological) heterogeneity, as shown, for example, in Fig. 3. Therefore, hunger can also stand as an idiopathic variable to reveal the particular strength of a drive in specific individuals. It is important not to confound a scientific approach with the promulgation of a common general law applicable to everyone on the planet. Science is, of course, concerned with underlying principles but it is also concerned with the description of individual uniqueness and variability; and with people acting under real-world conditions. This is exemplified by the use of smart phone applications and tracking technologies which now allow for the measurement of hunger and food intake under free-living conditions. These investigations show that individuals' eating patterns are very heterogeneous and erratic (Gill and Panda 2015); thus not following the conventional threemeal-per-day pattern. Understanding the underlying mechanisms of this individual heterogeneity in hunger and food intake may make it possible to use a personalized nutrition approach for the management of obesity and obesity-related diseases, for example.

Importantly, the scientific approach to hunger does not imply a simple mechanical idea that 'one model fits all'. In 
the context of psychology and medicine, it is common to see people behaving or responding differently to interventions or treatments; this is why group averages are misleading. This extreme inter-individual variability in response explains the difficulty in controlling an individual's hunger and food intake within the wider context of the treatment of obesity (UK Government's Foresight Programme 2007).

The issue of hunger in relation to the human problem of people living with obesity illustrates the tension between two explanatory forces underlying the scientific approach. One seeking to establish some general principles applicable to all; and the other paying respects to individual uniqueness. In this way, a modern approach to understanding still resonates with Allport's distinction between the nomothetic and the idiographic proposed more than 80 years ago (Allport 1937).

\section{Concluding Remarks}

Our aim in this manuscript has been to demonstrate how a scientific approach can lead to a true (or approximately true) understanding of hunger. This perspective is based on a validated measurement of hunger which reflects the intensity of a consciously perceived hunger sensation. This relies on the fact that hunger is a universal human experience. The existence of a valid, reliable, and widely agreed measurement of hunger allows researchers to investigate the mechanisms that give rise to the hunger sensation, and those which bring about its suppression. Such oscillations in perceived hunger are a common daily experience. A crucial feature of our thinking is that hunger can only be properly understood as part of a wider functional system which, for convenience, we have referred to as the appetite system. In turn, the reality of this situation has allowed hunger to be related to fundamental processes in human biology concerning the need for (food) energy to replenish energy used up in metabolic processes. This relationship between measured hunger and the biological system has clear functional value, and helps to explain the evolutionary value of hunger in maintaining life. In this way, the true (or approximately true) understanding of hunger helps us to explain a fundamental human experience.

Open Access This article is licensed under a Creative Commons Attribution 4.0 International License, which permits use, sharing, adaptation, distribution and reproduction in any medium or format, as long as you give appropriate credit to the original author(s) and the source, provide a link to the Creative Commons licence, and indicate if changes were made. The images or other third party material in this article are included in the article's Creative Commons licence, unless indicated otherwise in a credit line to the material. If material is not included in the article's Creative Commons licence and your intended use is not permitted by statutory regulation or exceeds the permitted use, you will need to obtain permission directly from the copyright holder. To view a copy of this licence, visit http://creativecommons.org/licenses/by/4.0/.

\section{References}

Allport GW (1937) Personality: a psychological interpretation. H Holt, New York

Beaulieu K, Hopkins M, Blundell J, Finlayson G (2018) Homeostatic and non-homeostatic appetite control along the spectrum of physical activity levels: an updated perspective. Physiol Behav 192:23-29

Beaulieu K, Hopkins M, Long C, Blundell JE, Finlayson G (2017) High habitual physical activity improves acute energy compensation in nonobese adults. Med Sci Sports Exerc 49(11):2268-2275

Berthoud HR, Munzberg H, Morrison CD (2017) Blaming the brain for obesity: integration of hedonic and homeostatic mechanisms. Gastroenterology 152(7):1728-1738

Blundell J, Finlayson G (2008) Mechanisms and biomarkers of appetite control. Agro Food Ind Hi-Tech 19(5 Suppl.):18-20

Blundell JE (1979) Hunger, appetite and satiety-constructs in search of identities. Nutrition and Lifestyles, Applied Science London, p. $21-42$.

Blundell JE (1991) Pharmacological approaches to appetite suppression. Trends Pharmacol Sci 12(4):147-157

Blundell JE, Caudwell P, Gibbons C, Hopkins M, Naslund E, King NA, Finlayson G (2012) Body composition and appetite: fat-free mass (but not fat mass or BMI) is positively associated with selfdetermined meal size and daily energy intake in humans. Br J Nutr 107(3):445-449

Blundell JE, de Graaf C, Hulshof T, Jebb S, Livingstone B, Lluch A, Mela D, Salah S, Schuring E, van der Knaap H, Westerterp M (2010) Appetite control: methodological aspects of the evaluation of foods. Obes Rev 11(3):251-270

Blundell JE, Gibbons C, Beaulieu K, Casanova N, Duarte C, Finlayson G, Stubbs RJ, Hopkins M (2020) The drive to eat in homo sapiens: energy expenditure drives energy intake. Physiol Behav 219:112846

Blundell JE, Gibbons C, Caudwell P, Finlayson G, Hopkins M (2015) Appetite control and energy balance: impact of exercise. Obes Rev 16:67-76

Blundell JE, Goodson S, Halford JC (2001) Regulation of appetite: role of leptin in signalling systems for drive and satiety. Int J Obes Relat Metab Disord 25(Suppl 1):S29-34

Blundell JE, Rogers PJ, Hill A (1987) Evaluating the satiating power of food: implications for acceptance and consumption. In: Solms JEA (ed) Food acceptance and nutrition. Academic Press, London, pp 205-219

Buckland NJ, Camidge D, Croden F, Lavin JH, Stubbs RJ, Hetherington MM, Blundell JE, Finlayson G (2018) A low energy-dense diet in the context of a weight-management program affects appetite control in overweight and obese women. J Nutri 148(5):798-806

Caudwell P, Finlayson G, Gibbons C, Hopkins M, King N, Naslund E, Blundell JE (2013) Resting metabolic rate is associated with hunger, self-determined meal size, and daily energy intake and may represent a marker for appetite. Am J Clin Nutr 97(1):7-14

Caudwell P, Gibbons C, Hopkins M, Naslund E, King N, Finlayson G, Blundell J (2011) The influence of physical activity on appetite control: an experimental system to understand the relationship between exercise-induced energy expenditure and energy intake. Proc Nutr Soc 70(2):171-180

Edholm OG, Adam JM, Healy MJ, Wolff HS, Goldsmith R, Best TW (1970) Food intake and energy expenditure of army recruits. Br J Nutr 24(4):1091-1107 
Edholm OG, Fletcher JG, Widdowson EM, McCance RA (1955) The energy expenditure and food intake of individual men. Br J Nutr 9(3):286-300

Erlanson-Albertsson C (2005) How palatable food disrupts appetite regulation. Basic Clin Pharmacol Toxicol 97(2):61-73

Finlayson G, King N, Blundell JE (2007) Liking vs. wanting food: importance for human appetite control and weight regulation. Neurosci Biobehav Rev 31(7):987-1002

Flint A, Raben A, Blundell JE, Astrup A (2000) Reproducibility, power and validity of visual analogue scales in assessment of appetite sensations in single test meal studies. Int J Obes Relat Metab Disord 24(1):38-48

Gibbons C, Caudwell P, Finlayson G, King N, Blundell J (2011) Validation of a new hand-held electronic data capture method for continuous monitoring of subjective appetite sensations. Int J Behav Nutr Phys Act 8:57

Gibbons C, Caudwell P, Finlayson G, Webb DL, Hellstrom PM, Naslund E, Blundell JE (2013) Comparison of postprandial profiles of ghrelin, active GLP-1, and total PYY to meals varying in fat and carbohydrate and their association with hunger and the phases of satiety. J Clin Endocrinol Metab 98(5):E847-855

Gibbons C, Finlayson G, Dalton M, Caudwell P, Blundell JE (2014) Metabolic phenotyping guidelines: studying eating behaviour in humans. J Endocrinol 222(2):G1-12

Gill S, Panda S (2015) A smartphone app reveals erratic diurnal eating patterns in humans that can be modulated for health benefits. Cell Metab 22(5):789-798

Hopkins M, Beaulieu K, Myers A, Gibbons C, Blundell JE (2017) Mechanisms responsible for homeostatic appetite control: theoretical advances and practical implications. Expert Rev Endocrinol Metab 12(6):401-415

Hopkins M, Duarte C, Beaulieu K, Finlayson G, Gibbons C, Johnstone AM, Whybrow S, Horgan GW, Blundell JE, Stubbs RJ (2019) Activity energy expenditure is an independent predictor of energy intake in humans. Int J Obes (Lond). https://doi.org/10.1038/ s41366-018-0308-6

Hopkins M, Finlayson G, Duarte C, Gibbons C, Johnstone AM, Whybrow S, Horgan GW, Blundell JE, Stubbs RJ (2018) Biological and psychological mediators of the relationships between fat mass, fat-free mass and energy intake. Int J Obes 43(2):233-242

Hopkins M, Finlayson G, Duarte C, Whybrow S, Ritz P, Horgan GW, Blundell JE, Stubbs RJ (2016) Modelling the associations between fat-free mass, resting metabolic rate and energy intake in the context of total energy balance. Int J Obes (Lond) 40(2):312-318

King NA, Caudwell PP, Hopkins M, Stubbs RJ, Naslund E, Blundell JE (2009) Dual-process action of exercise on appetite control: Increase in orexigenic drive but improvement in meal-induced satiety. Am J Clin Nutr 90(4):921-927

Lowe MR, Butryn ML (2007) Hedonic hunger: a new dimension of appetite? Physiol Behav 91(4):432-439

Mayer J, Roy P, Mitra KP (1956) Relation between caloric intake, body weight, and physical work: studies in an industrial male population in West Bengal. Am J Clin Nutr 4(2):169-175

Potochnik A (2017) Idealization and the aims of science. University of Chicago Press, Chicago, USA

Rolls BJ (2017) Dietary energy density: applying behavioural science to weight management. Nutr Bull 42(3):246-253

Saris WH, van Erp-Baart MA, Brouns F, Westerterp KR, ten Hoor F (1989) Study on food intake and energy expenditure during extreme sustained exercise: the Tour de France. Int J Sports Med 10(Suppl 1):S26-S31

Sokal A (2008) Beyond the Hoax: science, philosophy and culture. Oxford University Press, London

Stubbs RJ, Hughes DA, Johnstone AM, Rowley E, Reid C, Elia M, Stratton R, Delargy HJ, King N, Blundell JE (2000) The use of visual analogue scales to assess motivation to eat in human subjects: a review of their reliability and validity with an evaluation of new hand-held computerized systems for temporal tracking of appetite ratings. Br J Nutr 84(4):405-415

UK Government's Foresight Programme (2007). "Foresight. Tackling obesities: future choices - obesity system atlas." Retrieved from www.gov.uk/government/publications/reducing-obesity-obesitysystem-map.Accessed 5 Dec 2016

Publisher's Note Springer Nature remains neutral with regard to jurisdictional claims in published maps and institutional affiliations. 